\title{
Bare Printed Circuit Board Inspection Using Image Analysis
}

\author{
Divya C Thomas ${ }^{1}$, Devendra Sutar ${ }^{2}$, Jeetendra R Bhandankar ${ }^{3}$ \\ Electronics and Telecommunication Dept., Goa College of Engineering, Farmagudi, Ponda Goa, India ${ }^{1,2}$ \\ Senior Manager, Micro- Interconnecxion Pvt Ltd, Corlim, Goa, India ${ }^{3}$
}

\begin{abstract}
This paper reviews on a Bare Printed Circuit Board inspection system using image analysis wherein the inspection algorithm mainly focuses on the defect detection and classification using the real images. In this project, we have built a MATLAB program to detect Bare PCB faults and classify them and results are properly shown on a GUI using MATLAB. This paper reduces the flaws in defect detection using JIG tool and by human visual inspection. MATLAB image processing software combined with Arduino hardware provides a low cost solution for PCB inspection.
\end{abstract}

Keywords: PCB, MATLAB, Image Subtraction, Classification.

\section{INTRODUCTION}

Printed circuit board mechanically supports as well as electrically connects electronic components. PCB defect detection has great importance in the PCB manufacturing process. Human visual inspection is the traditional way to inspect any defect in PCB but it is time consuming as well as highly prone to errors due to human factors.

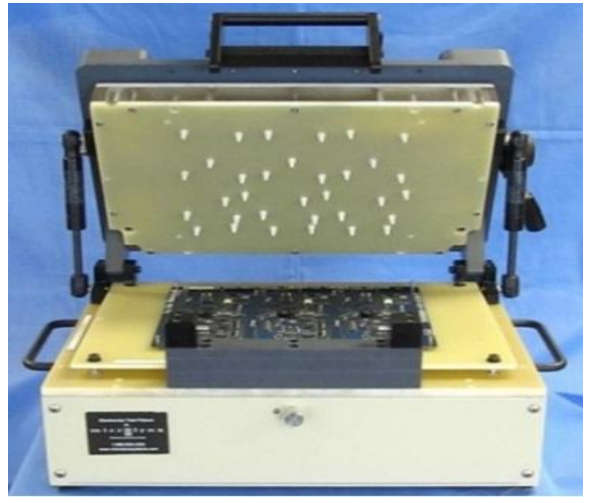

Fig 1. Jig tool

In the current process, the bare PCB testing is carried out using jig tool wherein connection between specific points are tested for short and open circuit. The output of the test indicates whether the PCB is faulty or not and test points where it is shorted or open. If found faulty then the bare PCB is visually inspected to find the type of fault.

Visual inspection is generally the most important part of PCB manufacturing. PCB inspection using image analysis can overcome the disadvantages of human inspection in case of fatigue, slowness and high cost. The advent of new electronic component fabrication technologies have made
PCB design complex and compact which causes difficulties to human inspection process thus increasing inspection duration. Therefore, an automated PCB inspection system can be implemented that provides real time analysis of the Bare PCB panels. In this paper we discuss a non-contact reference based image processing approach for defect detection and classification. A template of a defect free reference PCB image and a test PCB image are segmented and compared with each other using image subtraction and other procedures.

\section{BARE PCB DEFECTS}

[2]There are 14 known types of defects for single layer, bare PCBs.

\begin{tabular}{|l|l|}
\hline Sr. No. & Defects \\
\hline 1 & Breakout \\
\hline 2 & Pin Hole \\
\hline 3 & Open Circuit \\
\hline 4 & Under Etch \\
\hline 5 & Mouse Bite \\
\hline 6 & Missing Conductor \\
\hline 7 & Spur \\
\hline 8 & Short \\
\hline 9 & Wrong size hole \\
\hline 10 & Conductor too close \\
\hline 11 & Spurious copper \\
\hline 12 & Excessive short \\
\hline 13 & Missing hole \\
\hline 14 & Over etch \\
\hline
\end{tabular}




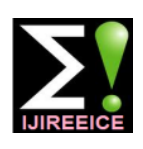

International Journal of Innovative Research in Electrical, Electronics, Instrumentation and Control Engineering

NCAEE 2017

National Conference on Advances in Electrical Engineering

NMAM Institute of Technology, Nitte

Vol. 5, Special Issue 2, April 2017

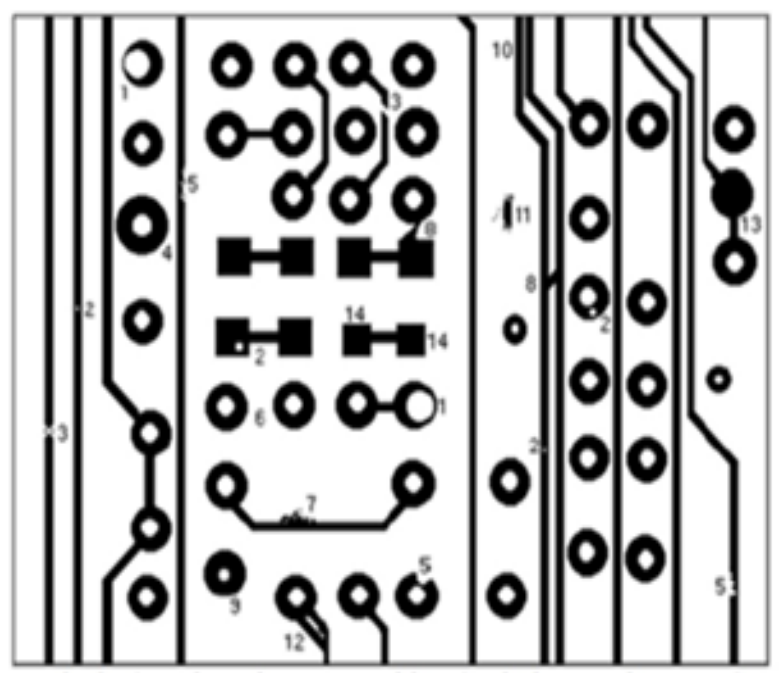

Fig 2. PCB defects

III. METHODOLOGY

This paper shows the method to automate the visual inspection of bare PCB to find the defect by image analysis using image processing tools. [4]This system is based on referential approach that has to be implemented on defected PCB and template images in order to detect number of defects present on bare PCB.

The project aims to find out the exact location of the defect on the PCB image by color differentiation and determine the type of defect.

\section{A. Overview}

Two images are needed for the inspection, the reference image and the test image. These images are captured from the reference and test PCB laminate using a highresolution camera. Hardware is designed to tackle problems such as misalignment of PCB, vertical distance at which the images are captured and images with bad illumination conditions.

Pre-processing of the image is done for obtaining accurate images which includes Image enhancement and Thresholding. Thresholding converts a gray scale image into a binary image for separation of objects of interest from the background so that further processing becomes easy.

Image subtraction operation is performed between the reference image and the test image in order to point out differences between them. By combining segmentation algorithm and image processing algorithm and using regional properties of elements in positive and negative images defect classification is done. The resultant image shows all the defects differentiated by pseudo-coloring and displayed on the GUI.
B. Defect Detection Algorithm

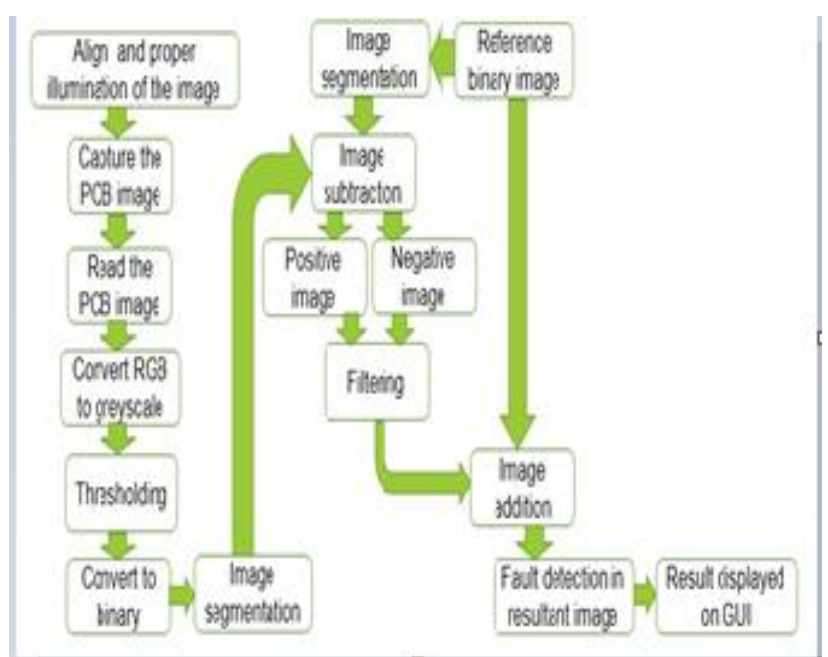

Fig 3. Algorithm

1) Image Acquisition:

[2] The process of selecting or capturing the image and giving it to system as an input is called image acquisition. Quality of the acquired image is one of key feature that depends on camera. When the camera devices are determined, it is crucial to provide proper illumination conditions to ensure image quality. Other factors such as orientation of the PCB and the height at which image is captured are considered for obtaining proper test and reference images by designing a proper hardware. Images captured by camera are in RGB format which has to be converted to gray scale for image enhancement and further processing.

\section{2) Image Preprocessing:}

Pre-processing of image is the most important step to increase accuracy of total processing procedure. Resizing the image according to desired needy area. After this it is necessary to enhance the edges. For enhancement we use histogram equalization. Because of enhancement, image shows edges and area becomes clear. Next step after this image enhancement of the image is thresholding.

[2] Thresholding is a method to convert a gray scale image into a binary image so that objects of interest are separated from the back ground. [2] Thresholding algorithm involves simply setting all pixel values to a value of 0 or 1 depending on whether the gray level at each pixel is greater than or less than a threshold.

The difficulty with this method is to select an optimal threshold value, especially when the area scan camera is used, it is very difficult to acquire images of uniform intensity due to the characteristics of the lighting source and camera lens. Binary test image is ready for image 
subtraction algorithm. Binary template image is dilated negative image are superimposed on the original reference according to the required tolerance so that some amount of image by pseudo-coloring in order to differentiate the extra copper is tolerable.

\section{3) Image Segmentation:}

[7] By combining the segmentation algorithm with the image processing algorithm five new images of segmented template and test images are produced.[7]When image subtraction is combined with the segmentation algorithm wherein each image is segmented into four patterns viz. hole segment, square segment, thin line and thick line, 20 new images are produced.

[7]In order to increase the number of groups and reduce number of defects in each group, image property measurement method is used to measure image regions after subtraction.

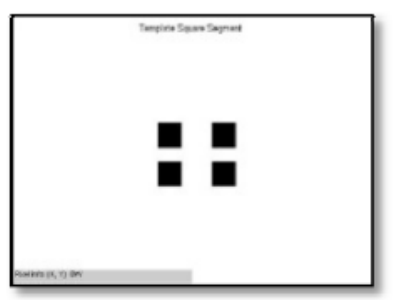

(a)Square segment

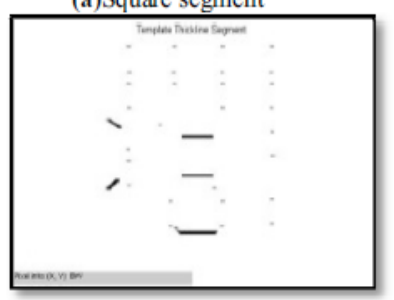

Fig 4. Image segments

4) Image Subtraction:

Image subtraction operation is performed in order to get the differences between two images that are the reference image and the inspected image and their segments. [2]The method compares both images pixel-by-pixel using XOR logic operator thus the resulting image obtained contains defects. [2]

The subtraction operation will produce negative and positive image, 1 represents white pixel and 0 represents black pixel in a binary image. Positive image is obtained by subtracting the test image from the reference image and negative image is the result of subtracting the reference image from the test image. Positive and negative images undergoes filtering in order to remove unwanted noise and obtain noise free images for further processing.

5) Fault Detection and Classification:

Open circuits or cuts in the PCB shown by positive image and short circuits or extra copper in the PCB shown by faults and find the exact location of fault. Using the image region properties and their measurements these defects are then classified. This image is then displayed on the GUI created and an error message will indicate the type of fault.

\section{IV.RESULTS}

\section{A. Capturing the PCB image}

GUI is created to capture the image and store in the database. Here the camera connected to the PC will start the preview after clicking 'Preview' button and stop the preview once the desired image is obtained by clicking 'stop preview' button. By selecting on 'store image' pushbutton the image will be stored in the Matlab database.

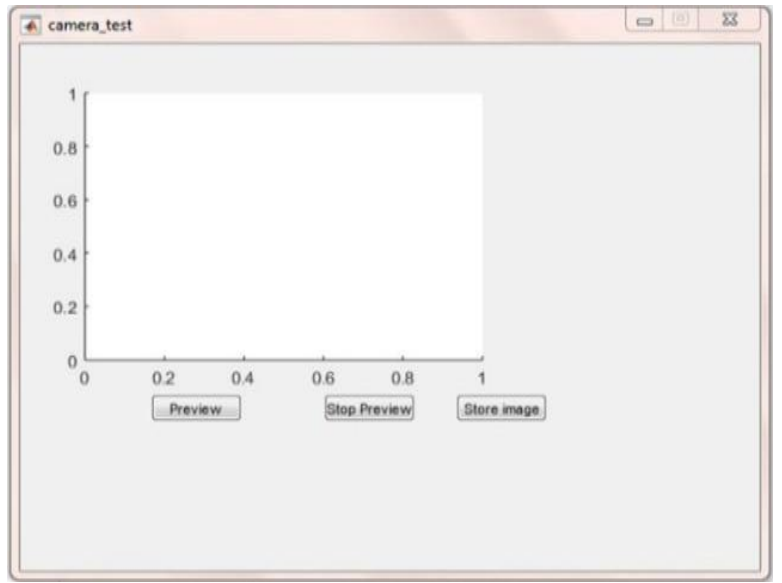

Fig 5. GUI for capturing image

B. Fault Detection and Classification

Test image and reference image is read from the database. The reference and test images in rgb format are converted to grayscale for easier further processing by thresholding. The reference and test grayscale images undergo image enhancement processes to obtain uniform brightness and sharpening the edges so that all the tracks are clear.

Reference image is dilated to the desired tolerance level entered by the user if some amount of extra copper is allowed. Next process is segmentation followed by subtraction of the reference and test images to obtain positive and negative images.

These images are then superimposed on the original rgb reference image to obtain the exact location of fault. An error message is displayed on textbox on the GUI specifying whether there is short or open circuit error or there is no fault and the type of fault. 

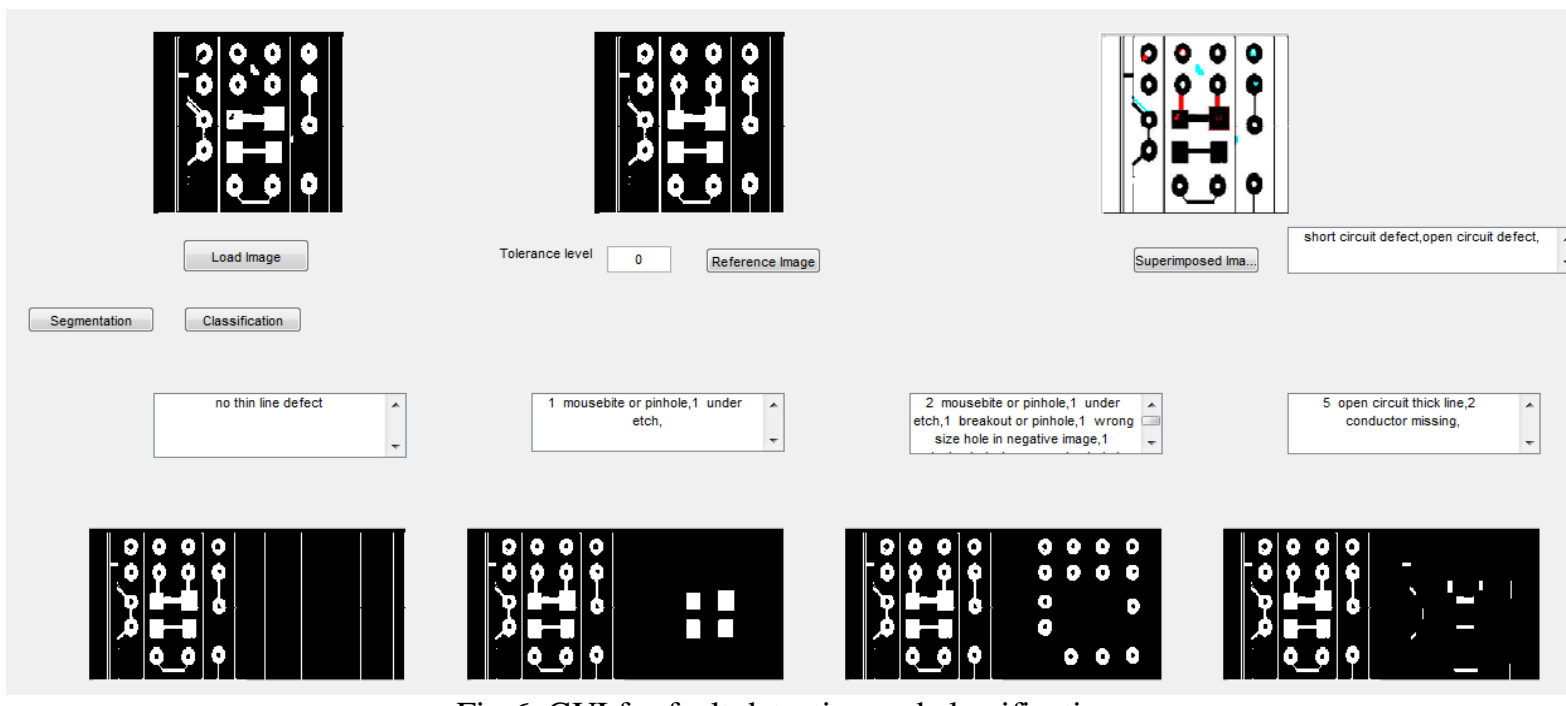

Fig 6. GUI for fault detection and classification

\section{CONCLUSION}

This paper shows an automated Bare Printed circuit Board inspection system for detecting defects by image analysis. While implementing the system, we need to make careful plans for each step such as image acquisition, image preprocessing, image subtraction and defect recognition.

At present, the finished designed inspection system can detect common PCB defects such as: short circuit, open circuit etc. in real time images. After images have been acquired, thresholding, noise filtering, segmentation and image subtraction operations are used to get noise-free positive and negative images which are then superimposed on the original reference image to obtain the exact position of the defect. In this system, the results are displayed on Matlab GUI as the user can load the image and start the processing on it by selecting given graphical elements such as pushbuttons for it. Also GUI is very simple to use as it does not need more experience or training to use.

\section{ACKNOWLEDGMENT}

I am deeply indebted to my guide, Prof. Devendra Sutar, Professor, Department of Electronics and Telecommunication Engineering, GEC and Mr. Jeetendra R Bhandankar, Senior Manager, MicroInterconnecxion Pvt Ltd., Corlim, Goa for allowing me to carry out this project under her supervision. He has given me confidence to take up this project and guided me at times of difficulty.

I thank my teachers, who have taught enthusiastically at the Goa College of Engineering. I also thank Dr. Hassanali Virani, (Head of the Department), Department of Electronics and Telecommunication Engineering and
Dr. Vinayak N. Shet (Principal) Goa College of Engineering for allowing me to do this project and providing the necessary infrastructure.

I thank my parents, family members and well-wishers, without whom this project would not have taken shape.

\section{REFERENCES}

[1] International Journal of Engineering and Innovative Technology (IJEIT) Volume 4, Issue 11, May 2015188

[2] 'A Review of PCB Defect Detection Using Image Processing', International Journal of Science, Engineering and Technology Research (IJSETR) Volume 4, Issue 4, April 2015 1. Anoop K.P, 2. Sarath N.S, 3. Sasi Kumar V. V.

[3] 'PCB INSPECTION SYSTEM USING IMAGE PROCESSING', International Conference on Computing, Communication and Automation (ICCCA2015) 2015 IEEE 11 1. Namita Kalyan Shinde, 2. Prof.S.S.Morade

[4] 'A Novel Approach of Standard Data Base Generation for Defect Detection in Bare PCB', International Journal of Innovative Research in Science, Engineering and Technology Vol. 5, Issue 6, June 20161. Mukesh Kumar, 2. Niraj Kumar Singh, 3. Manjesh kumar,4. Ajay kumar Vishwakarma

[5] 'PCB Fault Detection by Image Processing Tools: A Review', International Journal of Emerging Research in Management \&Technology (Volume-3, Issue-8) 1. Akash Kasturkar, 2. Dr.S. D. Lokhande Detection and Classification of Printed Circuit Board Defects Using Image Subtraction Method

[6] 1. Beant Kaur, 2. Gurmeet Kaur, 3. Amandeep Kaur 'MATLAB Based Defect Detection and Classification of Printed Circuit Board'Siti Hazurah Indera Putera,IEEE 2012 